\title{
Collisions between amorphous carbon nanoparticles: phase transformations
}

\author{
Maureen L. Nietiadi ${ }^{1}$, Felipe Valencia ${ }^{2,3}$, Rafael I. Gonzalez ${ }^{3,4}$, Eduardo M. Bringa ${ }^{4,5}$, and Herbert M. Urbassek ${ }^{1}$ \\ ${ }^{1}$ Physics Department and Research Center OPTIMAS, University Kaiserslautern, Erwin-Schrödinger-Straße, 67663 Kaiserslautern, \\ Germany \\ e-mail: urbassek@rhrk.uni-kl.de \\ 2 Centro de Investigación DAiTA Lab, Facultad de Estudios Interdisciplinarios, Universidad Mayor, Santiago, Chile \\ ${ }^{3}$ Center for the Development of Nanoscience and Nanotechnology CEDENNA, Avda. Ecuador 3493, Santiago 9170124, Chile \\ ${ }^{4}$ Centro de Nanotecnología Aplicada, Facultad de Ciencias, Universidad Mayor, Santiago 8580745, Chile \\ ${ }^{5}$ CONICET and Facultad de Ingeniería, Universidad de Mendoza, Mendoza 5500, Argentina
}

Received 16 April 2020 / Accepted 29 July 2020

\begin{abstract}
Context. Collisions of nanoparticles (NPs) occur in dust clouds and protoplanetary disks.

Aims. Sticking collisions lead to the growth of NPs, in contrast to bouncing or even fragmentation events and we aim to explore these processes in amorphous carbon NPs.

Methods. Using molecular-dynamics simulations, we studied central collisions between amorphous carbon NPs that had radii in the range of $6.5-20 \mathrm{~nm}$ and velocities of $100-3000 \mathrm{~m} \mathrm{~s}^{-1}$, and with varying $\mathrm{sp}^{3}$ content (20-55\%).

Results. We find that the collisions are always sticking. The contact radius formed surpasses the estimate provided by the traditional Johnson-Kendall-Roberts model, pointing at the dominant influence of attractive forces between the NPs. Plasticity occurs via sheartransformation zones. In addition, we find bond rearrangements in the collision zone. Low-sp ${ }^{3}$ material $\left(\mathrm{sp}^{3} \leq 40 \%\right)$ is compressed to $\mathrm{sp}^{3}>50 \%$. On the other hand, for the highest $\mathrm{sp}^{3}$ fraction, 55\%, graphitization starts in the collision zone leading to low-density and even porous material.

Conclusions. Collisions of amorphous carbon NPs lead to an increased porosity, atomic surface roughness, and changed hybridization that affect the mechanical and optical properties of the collided NPs.
\end{abstract}

Key words. planets and satellites: formation - protoplanetary disks - methods: numerical

\section{Introduction}

Besides silicates, $\mathrm{SiC}$, and carbonates, carbonaceous material is thought to form the bulk of interstellar dust grains (Draine 2003; Tielens 2005). This carbonaceous material may appear in various forms, including diamonds, graphite, amorphous carbon (a-C), hydrogenated amorphous carbon, polycyclic aromatic hydrocarbons (PAHs), and aliphatic hydrocarbons. Fullerenes are among the smallest carbonaceous grains found in the space environment (Dimitrijevic 2005; Tielens 2008; Micelotta et al. 2012), but the size distribution of the analogous PAC (polycyclic aromatic carbons) is expected to follow a slowly decaying powerlaw distribution (Mathis et al. 1977; Tielens 2005). The existence of diamonds may be the most interesting, since diamond is not the ground-state, but a high-pressure allotrope of carbon. Nanodiamonds are found in meteorites with mean sizes of around $2.6 \mathrm{~nm}$ (Lewis et al. 1989) and are thought to be of presolar origin due to the unusual $\mathrm{Xe}$ and Te isotopic ratios they contain. However, Dai et al. (2002) argue that nanodiamonds may have formed in the inner Solar System, since they are absent in comets. Nittler (2003) reviews stardust found in meteorites and also reports - besides diamond - graphitic contributions with larger sizes, 1-20 $\mu \mathrm{m}$. Vis et al. (2002) report besides diamond and graphitic structures, "black" carbon spheres, which may be amorphous or onions; in addition, hollow carbon structures - "sacs" or calabashes (Wang \& Yin 1998) - are observed, which may serve as containers of noble gases.
Nanodiamonds may have formed by a condensation process akin to chemical vapor deposition in space (Nuth 1987; Abdu et al. 2018), or by energetic collisions of precursor material. Thus, Tielens et al. (1987) argued that grain-grain collisions of graphitic carbon grains may lead to diamond formation, even though graphitic material may be rare (Wickramasinghe et al. 1999). The pertinent high collision velocities are attained, for instance, in the shock waves generated by supernova explosions, but also during the impact of asteroids on planets. While true carbon stars, in whose environment hydrocarbon molecules are abundant, are rare, stars like Elias 1 (V 892 Tau) are known to emit, besides PAHs, also nanodiamonds (Goto et al. 2009), which are thought to originate from initially graphitic material, onions for instance, under irradiation. This hypothesis was corroborated by molecular-dynamics simulations, which showed that collisions of carbon onions, which are multi-shell concentric fullerenes, may lead to diamond formation (Marks et al. 2012).

Molecular-dynamics (MD) simulations offer an intriguing route to study the consequences of nanoparticle (NP) collisions. In general, the collision behavior of NPs features a sticking regime at low velocities, followed by a bouncing regime where the kinetic collision energy allows the attractive surface forces to be surpassed; at even higher energies, strong energy dissipation by particle deformation and/or NP fragmentation again leads to the fusion of the colliding NPs (Kalweit \& Drikakis 2006; Ohnishi et al. 2008; Tanaka et al. 2012; Millán et al. 2016a,b; Nietiadi et al. 2019). It has been shown that silica nanoparticles 
will stick during collisions at velocities that are not too high (Nietiadi et al. 2017b), owing to their high surface energy; when the surface is passivated by $\mathrm{H}$ atoms, the bouncing velocity is reduced by a factor of almost an order of magnitude (Nietiadi et al. 2020). Ice NPs, on the other hand, will stick at all collision velocities, since at higher velocities collision-induced melting lets them readily deform and prevents bouncing (Nietiadi et al. 2017a). In an astrophysical context, NP sticking is relevant since it leads to mass agglomeration and thus dust growth (Dominik \& Tielens 1997).

Previous studies of carbonaceous NP collisions are rare. As mentioned above, Marks et al. (2012) showed diamond formation in the collision of carbon onions. Johnson et al. (2017) used ab-initio MD to study high-velocity $\left(10 \mathrm{~km} \mathrm{~s}^{-1}\right)$ collisions of diamond NPs resulting in NP fragmentation. Sun et al. (2015) used a rigid force field, in which the carbon conformation cannot change, to study energetic impacts of diamond and also of amorphous carbon (a-C) NPs; due to their use of a rigid force field, they could not detect phase transformations. Recently they extended their simulations using a more flexible potential (Tersoff 1988) and reported that in the velocity regime of $1-2 \mathrm{~km} \mathrm{~s}^{-1}$, a-C NPs of a radius of 2-5 nm will bounce (Sun et al. 2020).

An understanding of the mechanical properties of a-C NPs is also relevant in other areas of science and technology. Liu et al. (2017) demonstrate that a-C nano-pillars show both high yield strength and large plasticity, which is interesting for their application in micro and/or nano-electro-mechanical systems (MEMS/NEMS; Suk et al. 2012). In addition, the combination of strength and flexibility (Yang et al. 2016, 2018) of amorphous, and also porous, carbon NPs helps to overcome the characteristic lack of ductility shown by a-C-based materials. These features are relevant for converting a-C NPs into promising candidates for protective coatings and also for the design of ( $\mathrm{H}$ and $\mathrm{Li}$ ) energy storage devices (Panella et al. 2005; Zhang et al. 2014).

In the present paper, we study energetic collisions between a-C NPs. Such NPs might easily have originated from the condensation of C-rich atmospheres around stars by the chemical-vapor-deposition processes mentioned above. The science of a-C is interesting since a continuum of variants exists that differ in the fraction of $\mathrm{C}$ atoms bonded in $\mathrm{sp}^{2}$ or $\mathrm{sp}^{3}$ bonds. We therefore create a-C specimens with a $\mathrm{sp}^{3}$ fraction between 20 and $55 \%$. We study how energetic collisions between such a-C NPs change the $\mathrm{sp}^{3}$ fraction and hence the physical and chemical properties of the NPs.

For computational reasons, our study focuses on small nanometer-sized grains with radii $\leq 20 \mathrm{~nm}$, and on central collisions. It is therefore not comprehensive, but aims at showing which phenomena show up in this collision system that have not been encountered before in the better understood silica-grain collisions. We thus direct attention to the phase transformations induced in carbon-grain collisions that alter the mechanical properties (elastic moduli and surface energies) of the grains. These alterations need to be taken into account when attempting to set up a database to be used to model the size evolution of carbon dust clouds, similar to the work of Ormel et al. (2009) for ice and silica grains. Since the $\mathrm{sp}^{3}$ content also influences the optical properties of NPs (Koike et al. 1980; Zubko et al. 1996; Hiratsuka et al. 2013), the collision history of grains influences their refractive indices and extinction coefficients.

\section{Method}

There are many interatomic interaction potentials to describe the $\mathrm{C}-\mathrm{C}$ interaction, as described, for instance, in the comparative A159, page 2 of 9
Table 1. Fraction of $\mathrm{sp}^{3}$-hybridized atoms, $\mathrm{sp}^{3}$, mass density $\rho$, average coordination $Z$, Young's modulus $E$, specific surface energy $\gamma$, velocity of sound $c_{\mathrm{s}}$, and collision pressure $p$ for a $v=600 \mathrm{~m} \mathrm{~s}^{-1}$ collision (Eq. (4)) for the simulated a-C samples.

\begin{tabular}{rrrrrrr}
\hline \hline $\begin{array}{r}\mathrm{sp}^{3} \\
(\%)\end{array}$ & $\begin{array}{c}\rho \\
\left(\mathrm{g} \mathrm{cm}^{-3}\right)\end{array}$ & $\langle Z\rangle$ & $\begin{array}{c}E \\
(\mathrm{GPa})\end{array}$ & $\begin{array}{c}\gamma \\
\left(\mathrm{J} \mathrm{m}^{-2}\right)\end{array}$ & $\begin{array}{c}c_{\mathrm{s}} \\
\left(\mathrm{km} \mathrm{s}^{-1}\right)\end{array}$ & $\begin{array}{c}p \\
(\mathrm{GPa})\end{array}$ \\
\hline 20 & 2.15 & 2.94 & 195.4 & 1.78 & 9.53 & 12.3 \\
30 & 2.46 & 3.20 & 304.6 & 2.00 & 11.13 & 16.4 \\
40 & 2.71 & 3.38 & 396.1 & 2.16 & 12.09 & 19.7 \\
55 & 3.07 & 3.53 & 561.2 & 2.44 & 13.52 & 24.9 \\
\hline
\end{tabular}

reviews by de Tomas et al. (2016, 2019). Here we used the environment-dependent interaction potential (EDIP) for carbon designed by Marks (2000). It is well suited to describe the diamond-graphite transition, and was shown to fare well in describing a-C (de Tomas et al. 2016, 2019).

The a-C samples are prepared by quenching liquid carbon, based on the procedures of Marks et al. (2002) and de Tomas et al. (2016). A total of $473344 \mathrm{C}$ atoms are ordered in a simple cubic structure in a $82 a_{0} \times 82 a_{0} \times 42 a_{0}$ supercell with a lattice parameter $a_{0}$. The lattice constant $a_{0}$ is treated as a free parameter to control the a-C density. The crystal is quickly heated to $5000 \mathrm{~K}$ with a heating rate of $10^{4} \mathrm{~K} \mathrm{ps}^{-1}$ by means of a velocity scaling algorithm. Then the liquid carbon is quenched from 5000 to $300 \mathrm{~K}$ in $0.5 \mathrm{ps}$ using an exponential cooling rate. The fast cooling rate ensures that the liquid carbon solidifies in an amorphous structure. Finally, the sample is kept at $300 \mathrm{~K}$ for 50 ps to allow system relaxation and bond rearrangements. The $\mathrm{sp}^{3}$ content of each sample is characterized using the pair correlation function with a cut off of $2.0 \AA$. The a-C specimens contain $\mathrm{sp}^{3}$ fractions of $\mathrm{sp}^{3}=20,30,40$, and $55 \%$, and we denote the $\mathrm{sp}^{3}$ fraction in our specimen by $\mathrm{sp}^{3}$. The densities obtained (see Table 1) are in good agreement with experimental results and density-function-theory calculations (Schwan et al. 1996; Marks et al. 2002; de Tomas et al. 2016, 2019). We note that in previous studies, several many-body potentials have been employed for a$\mathrm{C}$ modeling; however, not all of them have demonstrated good results in reproducing the a-C density, microstructure, and the $\mathrm{sp}^{3}$ content simultaneously ( $\mathrm{Li}$ et al. 2013a, 2018). However, the EDIP potential, with the quenching method employed here, has been shown to give reliable results if compared to experimental evidence and first-principles calculations (Marks et al. 2002).

Young's modulus, $E$, is an important parameter to describe the collision mechanics; we determine it from a uniaxial loading simulation at a strain rate of $1.0 \times 10^{-8} \mathrm{~s}^{-1}$ up to a final strain of 0.01 . In general, for a-C, the dependence of $E$ on $\mathrm{sp}^{3}$ is highly sensitive to the interatomic potential (Jana et al. 2019). From the so-called constraint model of covalent glasses proposed by He \& Thorpe (1985), we know that the a-C Young's modulus increases with the average coordination $\langle Z\rangle$ as

$E=k\left(\langle Z\rangle-Z_{0}\right)^{n}$.

Here, $Z_{0}$ marks the transition from a floppy to a rigid network and $k$ and $n$ are fit parameters. We determine $\langle Z\rangle$ from the atomistic data; our results (see Table 1) successfully fulfill the dependence $\langle Z\rangle=3+\mathrm{sp}^{3}$ predicted by Ferrari et al. (1999b), in particular for higher $\mathrm{sp}^{3}$ fractions.

Our a-C specimens fulfill Eq. (1) with fit factors $Z_{0}=2.4$, $k=443.69 \mathrm{GPa}$, and $n=1.54$, in good agreement with the value $n=1.5$ of the model (He \& Thorpe 1985). Besides, our EDIP results extrapolate to a Young's modulus of $915 \mathrm{GPa}$ for a fully 
Table 2. Number $N$ of $\mathrm{C}$ atoms in a NP of radius $R$ and $\mathrm{sp}^{3}$ fraction $\mathrm{sp}^{3}$.

\begin{tabular}{rrrrr}
\hline \hline$R(\mathrm{~nm})$ & $\mathrm{sp}^{3}=20 \%$ & $\mathrm{sp}^{3}=30 \%$ & $\mathrm{sp}^{3}=40 \%$ & $\mathrm{sp}^{3}=55 \%$ \\
\hline 6.5 & 123380 & 142068 & 156101 & 173031 \\
10 & 449333 & 517423 & 568346 & 630104 \\
15 & 1517403 & 1747037 & 1917716 & 2126449 \\
20 & 3596781 & 4141658 & 4545089 & 5040403 \\
\hline
\end{tabular}

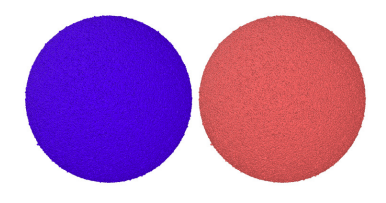

(a)

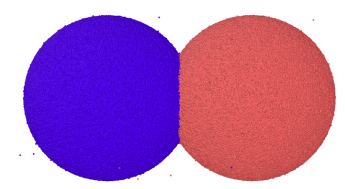

(b)
Fig. 1. Snapshots showing the $(a)$ initial and $(b)$ final conformation of two $R=20 \mathrm{~nm} \mathrm{NPs}, \mathrm{sp}^{3}=20 \%$ at a velocity of $v=600 \mathrm{~m} \mathrm{~s}^{-1}$. Atoms are colored according to their original grain affiliation.

coordinated network $(\langle Z\rangle=4)$, which is only slightly smaller than that of diamond $(\sim 1000 \mathrm{GPa})$, as expected due to the amorphous structure of a-C. Additionally, the model achieves good agreement with the available experimental results of tetrahedral a-C (Kelires 1994; Ferrari et al. 1999b; Charitidis 2010).

The specific surface energies $\gamma$ were calculated by the usual procedure (Wolf 1990), which consists in comparing the potential energy of bulk material with that of material containing a planar surface. Our values (see Table 1) show a clear increase of $\gamma$ with $\mathrm{sp}^{3}$ fraction. As a comparison, for graphite it is $\gamma=$ $0.1 \mathrm{~J} \mathrm{~m}^{-2}$ (Li et al. 2013b), while in diamond - depending on the surface orientation $-\gamma$ may reach $4 \mathrm{~J} \mathrm{~m}^{-2}$ (Kern \& Hafner 1997; De La Pierre et al. 2014). This strong dependence on $\mathrm{sp}^{3}$ fraction is in line with the notion that available dangling bonds at a surface increase the specific surface energy. We note that surface passivation would strongly change $\gamma$; in the comparable case of silica NPs, surface passivation lets the surface energy decrease from 1.43 to $0.08 \mathrm{~J} \mathrm{~m}^{-2}$ (Nietiadi et al. 2017b, 2020).

For preparation of the NPs, we replicate the cuboid samples and cut out spheres of a predefined radius $R$. For radius $R=6.5$ $(10,15,20) \mathrm{nm}$, each NP contained between $N=123,380$ and $5040403 \mathrm{C}$ atoms; the exact numbers for each $\mathrm{sp}^{3}$ fraction are provided in Table 2. The spheres are relaxed for $50 \mathrm{ps}$ in an NPT environment, cooling their temperatures to below $1 \mathrm{~K}$, and relaxing the internal pressure to zero. Since during this process the NP surface exhibited atomistic roughness, atoms sticking out by more than $3.4 \AA$ were removed.

To perform collision simulations, the NPs were copied and put at a distance of $2 \mathrm{~nm}$, outside the cut-off radius of the potential (see Fig. 1a). Then the two NPs were given a relative velocity $v$ to start the collision. Only central (head-on) collisions were considered. The simulations were followed for 40 ps. The MD simulations were performed with the LAMMPS code (Plimpton 1995). Atomistic snapshots were generated with OVITO (Stukowski 2010).

\section{Results}

\subsection{Contact radius}

In all cases studied - radii up to $R=20 \mathrm{~nm}$, velocities between 100 and $3000 \mathrm{~m} \mathrm{~s}^{-1}$, and for all $\mathrm{sp}^{3}$ fractions - we only observed

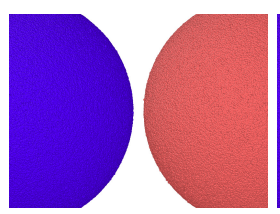

(a)

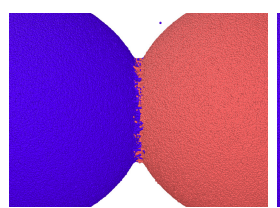

(d)

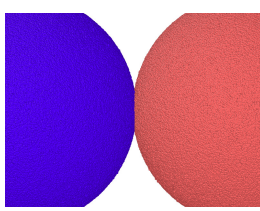

(b)

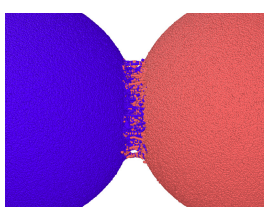

(e)

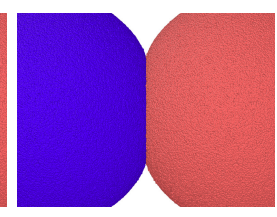

(c)

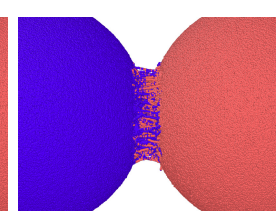

(f)
Fig. 2. Snapshots showing a collision of two $R=20 \mathrm{~nm} \mathrm{NPs}, \mathrm{sp}^{3}=$ $55 \%$ at a velocity of $v=600 \mathrm{~m} \mathrm{~s}^{-1}$, at $(a)$ the start of the simulation, $0 \mathrm{ps},(b)$ jump to contact, $2 \mathrm{ps},(c)$ the moment of maximum compression, $8 \mathrm{ps}$, and during the expansion regime at times of $(d) 15 \mathrm{ps}$, (e) $20 \mathrm{ps}$, and $(f) 40 \mathrm{ps}$. Atoms are colored according to their original grain affiliation.

sticking; the two NPs never bounced off each other. A typical result of the sticking is shown in Fig. $1 \mathrm{~b}$ for the specific case of $\mathrm{sp}^{3}=20 \%, R=20 \mathrm{~nm}$, and $v=600 \mathrm{~m} \mathrm{~s}^{-1}$. Due to the high collision energy, a few weakly bound $\mathrm{C}$ atoms are shattered off the surface. In the contact zone, some intermixing of grain material can be observed on an atomic scale. The radius of the approximately circular contact zone is denoted as the contact radius, $a_{c}$; it is here approximately $11.8 \mathrm{~nm}$, around $60 \%$ of the NP radius $R$.

For the $\mathrm{sp}^{3}=55 \% \mathrm{NPs}$, the same collision conditions lead to a quite different contact zone (see Fig. 2). During the compressive phase of the collision (Figs. 2b and c) the contact looks similar to the $\mathrm{sp}^{3}=20 \%$ case (Fig. 2). However, later, when the two NPs expand again and attempt to lose their contact, the contact zone expands along the collision apsis, but the two NPs stay connected by a multitude of filaments. These mostly consist of $\mathrm{C}$ material stemming from a single grain that is elongated along the tearing direction. This is the only case observed by us that comes close to bouncing; we note, however, that the NPs never separate. We checked the collision zone in particular for velocities between 200 and $800 \mathrm{~m} \mathrm{~s}^{-1}$; in all these cases the filamentary fuzzy structure developed, as in Fig. 2; of course, in accordance with the increase of the contact radius with velocity, the radial size of this filamentary structure also increased.

Similar monoatomic filaments were observed in previous simulations of silica NP collisions (Nietiadi et al. 2017b). For that material, however, the filaments could rupture at sufficiently high collision velocities. In C material, diamond NP collisions (Johnson et al. 2017), tension tests of C nanotubes (Liew et al. 2004), and the fracture of graphene grain boundaries (Zhang et al. 2013) led to monoatomic filaments similar to the ones observed here.

The dynamics of the contact is given - besides the contact radius - by the time evolution of the overlap $\delta$. It is defined by the difference of the distance $d$ between the centers of the two NPs and $2 R, \delta=2 R-d$; positive overlap thus means a compressive deformation of the two NPs. In Fig. 3 we plot the overlap and contact radius of the two collisions shown in the snapshots of Figs. 1 and 2. During the early part of the compressive phase until time $t=5 \mathrm{ps}$ with overlaps of around $4 \mathrm{~nm}$ and contact radii of $8 \mathrm{~nm}$ - both collisions are quite similar. However, later significant differences show up. The $\mathrm{sp}^{3}=20 \%$ case leads to further compression with a maximum at $11 \mathrm{ps}$ where $\delta$ reaches almost $8 \mathrm{~nm}$, followed by a series of oscillations of the collided NP pair, 


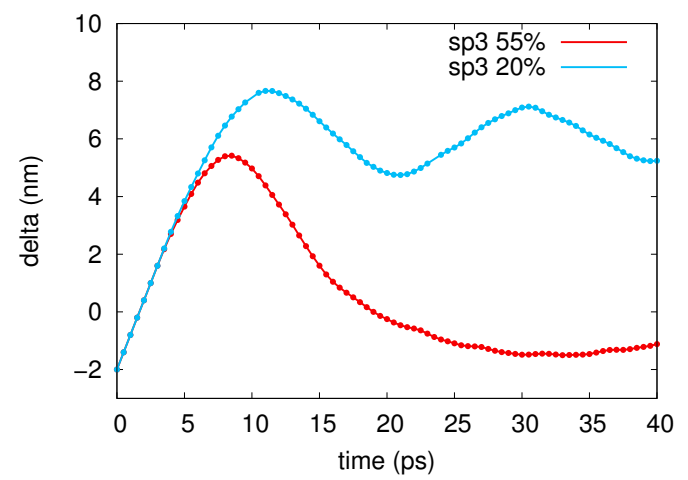

(a)

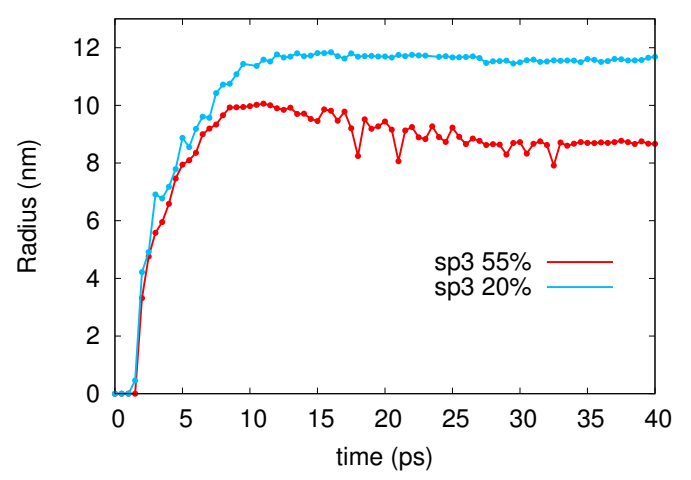

(b)

Fig. 3. Time evolution of the (a) overlap, $\delta$, and $(b)$ contact radius, $a_{c}$, in the collision of two NPs of radius $R=20 \mathrm{~nm}$ colliding with velocity $v=600 \mathrm{~m} \mathrm{~s}^{-1}$. Data are for two $\mathrm{sp}^{3}$ fractions, $\mathrm{sp}^{3}=20$ and $55 \%$.

which slowly dampens its amplitude. The contact radius stabilizes its size of $11.8 \mathrm{~nm}$ after the time of strongest depression; even during the NP oscillations the contact radius stays constant, thus proving the attractive strength of the contact.

The $\mathrm{sp}^{3}=55 \%$ case shows a smaller maximum overlap of only $5 \mathrm{~nm}$, which points at a larger stiffness of the $\mathrm{sp}^{3}=55 \%$ a-C material. During the ensuing expansion phase, the overlap reaches negative values after around $18 \mathrm{ps}$; this means that the NPs are farther apart than $2 R$; this is the phase where the filamented neck forms between the two NPs, which is seen in Figs. 2e and f. The buildup of the neck dampens the NP oscillations. The contact radius does not quite reach the size of the $\mathrm{sp}^{3}=20 \%$ case and has a maximum of $10 \mathrm{~nm}$; it slowly decreases to only $8.5 \mathrm{~nm}$ while the overlap becomes negative.

In Fig. 4, we investigate to what extent the collision velocity influences the features described in Fig. 3b. It is observed that with increasing collision velocity, the contact radius increases (see Fig. 4) but the features mentioned above, remain analogous.

The time dependence of the contact radius may be further analyzed by appealing to the Johnson-Kendall-Roberts (JKR, Johnson et al. 1971) theory of adhesive contacts. It predicts a relation between the overlap $\delta$ and the contact radius $a_{c}-$ which we denote here as $a_{\mathrm{JKR}}$ to emphasize its derivation from JKR theory - as (Maugis 2000; Brilliantov et al. 2007)

$\delta=\frac{2 a_{\mathrm{JKR}}^{2}}{R}-\sqrt{8 \pi \frac{\gamma a_{\mathrm{JKR}}}{E_{\mathrm{ind}}}}$.

Here, $\gamma$ is the specific surface energy and $E_{\text {ind }}=E /\left(1-v^{2}\right)$ is the indentation modulus, where $E$ is Young's modulus and $v$ is the Poisson ratio. As the influence of $v$ is small, we set $E_{\text {ind }} \simeq E$.

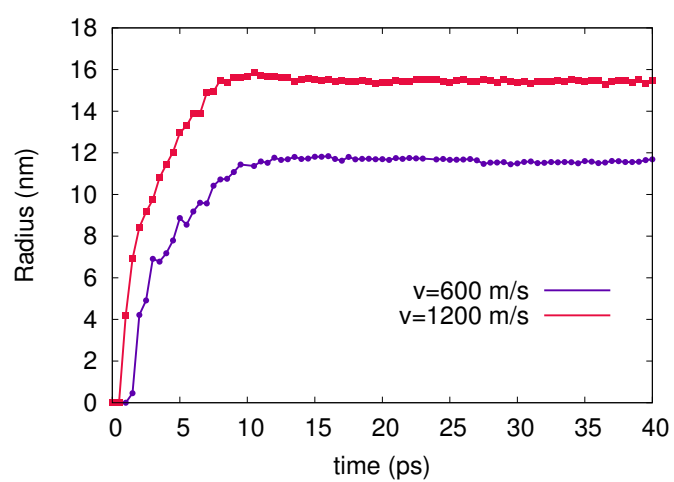

(a)

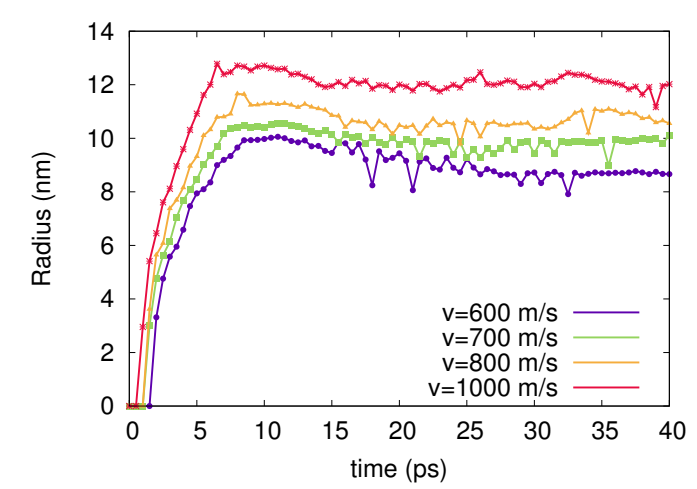

(b)

Fig. 4. Time evolution of the contact radius, $a_{c}$, in the collision of two NPs of radius $R=20 \mathrm{~nm}$, for an $\mathrm{sp}^{3}$ fraction of $(a) \mathrm{sp}^{3}=20 \%$ and (b) $\mathrm{sp}^{3}=55 \%$, colliding with various velocities $v$.

We use Eq. (2) to determine from our data of $\delta(t)$ a corresponding contact radius via Eq. (2), which we denote as $a_{\mathrm{JKR}}$, and compare to our simulated data of the contact radius, $a_{c}$ (see Fig. 5). Indeed, the rising part of the contact radius is quite well reproduced by the JKR theory. However, the contact radius at maximum compression is underestimated, and the behavior during the oscillatory motion in the $\mathrm{sp}^{3}=20 \%$ case, and even more during the NP expansion in the $\mathrm{sp}^{3}=55 \%$ case, is not reproduced at all. This shows that the role of the attractive forces between the two NPs is not well reproduced by JKR theory. A similar failure of JKR was noticed previously in the collision of silica NPs (Nietiadi et al. 2017b), and even more so in the collision of ice NPs (Nietiadi et al. 2017a).

Johnson-Kendall-Roberts theory also allows us to extract a prediction of the bouncing velocity, $v_{b}$, which is the smallest velocity at which two NPs bounce. It is given by (Thornton \& Ning 1998; Brilliantov et al. 2007; Krijt et al. 2013)

$v_{b}=\left(\frac{C}{\rho}\right)^{1 / 2}\left(\frac{\gamma^{5}}{E_{\text {ind }}^{2} R^{5}}\right)^{1 / 6}$.

Here, $\rho$ is the mass density and $C$ is a constant that incorporates dissipative processes during the collision. A wide range of values - from $C=5.25$ (Brilliantov et al. 2007) over 18.3 (Dominik \& Tielens 1997) and 57.9 (Kimura et al. 2015) to 669 (Nietiadi et al. 2017b) - has been used for this constant. Using the highest value of $C$, the bouncing velocity of $R=20 \mathrm{~nm}$ spheres is predicted by Eq. (3) to be between $776 \mathrm{~m} \mathrm{~s}^{-1}$ for sp $\mathrm{sp}^{3}=20 \%$ and $595 \mathrm{~m} \mathrm{~s}^{-1}$ for $\mathrm{sp}^{3}=55 \%$. The mild decrease with $\mathrm{sp}^{3}$ content is due to the fact that the surface energy only increases weakly, 
M. L. Nietiadi et al.: Collisions between amorphous carbon nanoparticles

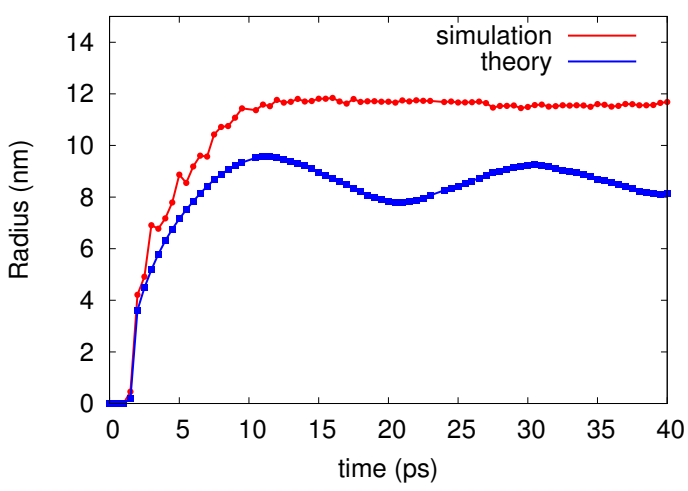

(a)

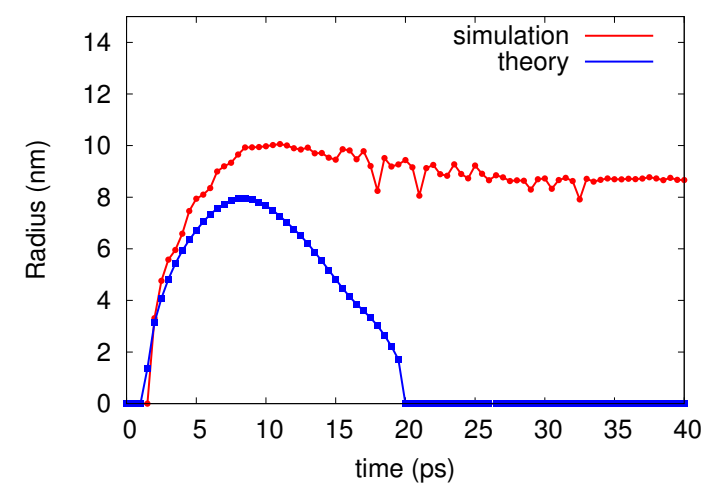

(b)

Fig. 5. Time evolution of the contact radius, $a_{c}$, in the collision of two NPs of radius $R=20 \mathrm{~nm}$, colliding with velocity $v=600 \mathrm{~m} \mathrm{~s}^{-1}$ for $\mathrm{sp}^{3}$ fractions of $(a) \mathrm{sp}^{3}=20 \%$ and $(b) \mathrm{sp}^{3}=55 \%$. The simulation results are compared to the estimate of JKR theory, Eq. (2).

while both density and elastic modulus increase more strongly with $\mathrm{sp}^{3}$ content (see Table 1).

In our simulations, we do not find bouncing for these velocities (nor for any other velocity in the range investigated). However, in the velocity range of $600-800 \mathrm{~m} \mathrm{~s}^{-1}$, we do find the formation of the filamentary adhesive neck (see Fig. 2), which is induced when during the attempted separation of the two NPs after the collision, hybridization changes in the bonding prevent bouncing. So the velocity regime of $600-800 \mathrm{~m} \mathrm{~s}^{-1}$ comes closest to the bouncing case. We conclude that the role of attractive forces in the collision of a-C NPs is larger than anticipated in macroscopic theories, like JKR or even the Derjaguin-MullerToporov (DMT) model (Derjaguin et al. 1975), where attractive van-der-Waals forces are included. Therefore, nanoscopic a-C NPs do not bounce.

\subsection{Collision-induced phase transformation}

We monitor the bonding in the collision zone in the final state by the $\mathrm{sp}^{3}$ fraction. To be specific, besides an analysis in the entire system, we performed the analysis in a slab centered in the collision plane (mid-plane between the two spheres, perpendicular to the collision apsis) with a fixed width of $4 \mathrm{~nm}$, thus extending $2 \mathrm{~nm}$ into each of the two colliding NPs. We note that the grain-averaged data show the same trends as the data obtained in the slab, but naturally exhibit smaller collisioninduced changes. Atoms are classified according to the number of nearest neighbors, $Z$; atoms with $Z=2(3,4)$ are $\mathrm{sp}^{1}\left(\mathrm{sp}^{2}, \mathrm{sp}^{3}\right)$ bonded.

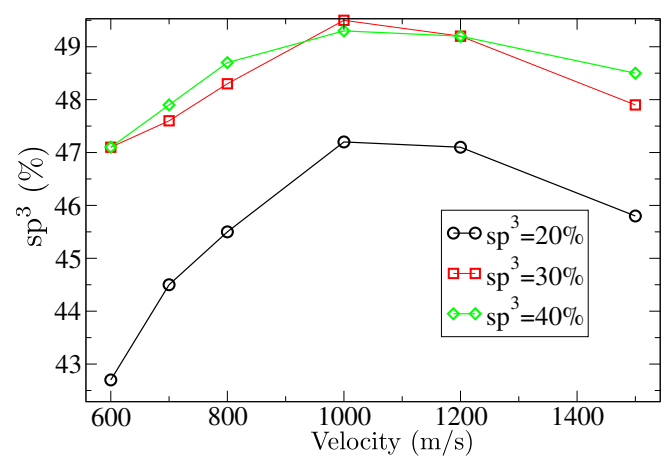

(a)

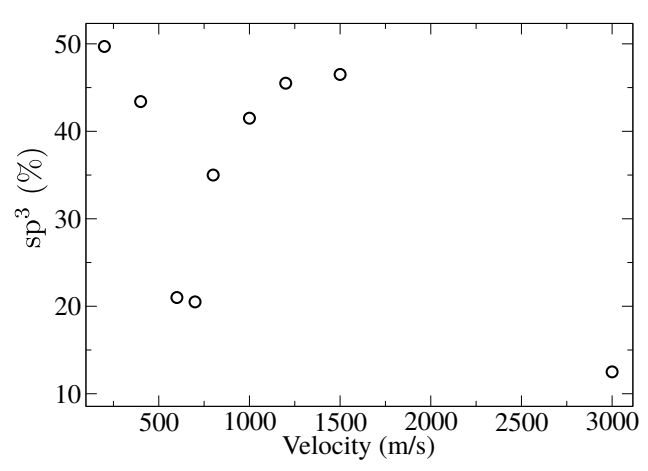

(b)

Fig. 6. Dependence of $\mathrm{sp}^{3}$ fraction in the contact region on collision velocity $v$. Data are for $R=20 \mathrm{~nm}$ NPs with $(a)$ various initial $\mathrm{sp}^{3}$ fractions (see legend) and $(b)$ for $\mathrm{sp}^{3}=55 \%$.

During the collision, the $\mathrm{sp}^{3}$ content significantly changes. Figure 6 shows the changes in the collision zone for $R=20 \mathrm{~nm}$ $\mathrm{NPs}$ as a function of collision velocity. For low $\mathrm{sp}^{3}$ content $\left(\mathrm{sp}^{3}=20-40 \%\right)$, Fig. 6a shows an increase of $\mathrm{sp}^{3}$ content for velocities below $1000 \mathrm{~m} \mathrm{~s}^{-1}$. In the collision zone, the $\mathrm{sp}^{3}$ fraction increased up to almost 50-55\%. This process is akin to the phase transformation from graphite to diamond occurring at high pressures and is caused by the collision-induced compression, which increases the number of nearest neighbors, and hence the $\mathrm{sp}^{3}$ fraction.

For larger velocities, the $\mathrm{sp}^{3}$ fraction decreases again. We presume that this is caused by the high temperature building up in the collision zone. It is known that for temperatures above around 800-900 K, a-C shows a stress release and softening that is accompanied by bond rearrangements (Ferrari et al. 1999a). Indeed our simulations show that temperatures increase with velocity in the collision zone and reach values above this threshold (see Sect. 3.3 below). In addition to these thermal effects, at higher velocities more energy is lost to elastic waves that are emitted from the contact zone so the energy density at the interface is lower when integrated over the few picoseconds where compaction occurs. We note that when averaged over the entire system, the $\mathrm{sp}^{3}$ fraction shows only a mild, but continuous, increase with velocity, from 21 to $30 \%$ for the $\mathrm{sp}^{3}=20 \%$ system, and from 41 to $44 \%$ for the $\mathrm{sp}^{3}=40 \%$ NPs.

For the $\mathrm{sp}^{3}=55 \%$ NPs, however, the collision reduces the $\mathrm{sp}^{3}$ fraction (see Fig. 6b). Since this phenomenon is opposite to that found in the $\mathrm{sp}^{3}$-poor samples, we increased the velocity range where we monitored the collision zone for this case. 

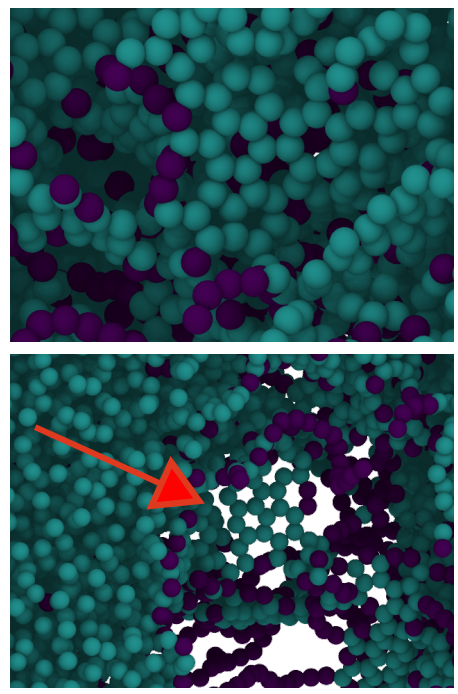

Fig. 7. Graphitic structures generated in the collision zone of two $R=$ $20 \mathrm{~nm} \mathrm{NPs} \mathrm{of} \mathrm{sp}^{3}=55 \%$ at velocity $v=800 \mathrm{~m} \mathrm{~s}^{-1}$. Atoms are colored according to their bonding configuration: green $\left(\mathrm{sp}^{2}\right)$, blue $\left(\mathrm{sp}^{1}\right)$.

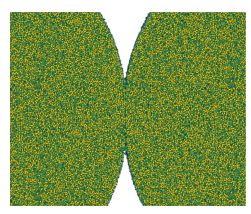

$\mathrm{v}=100 \mathrm{~m} / \mathrm{s}$

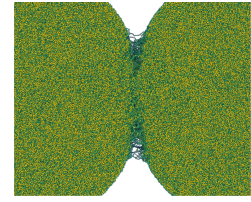

$\mathrm{v}=800 \mathrm{~m} / \mathrm{s}$

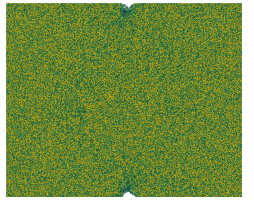

$v=1500 \mathrm{~m} / \mathrm{s}$ sp1

Fig. 8. Snapshots showing the final conformation of two $R=20 \mathrm{~nm}$ NPs, $\mathrm{sp}^{3}=55 \%$, at the collision velocities indicated. Atoms are colored according to their bonding configuration.

At small velocities, $v \leq 700 \mathrm{~m} \mathrm{~s}^{-1}$, the $\mathrm{sp}^{3}$ fraction continually decreases. As discussed in Sect. 3.1, the origin of the low $\mathrm{sp}^{3}$ fraction in the collision zone is the adhesive glue-like zone arising from the attractive forces holding the two NPs together (see Fig. 2 above and its discussion). Indeed, a closer look at the collision zone shows graphitic structures that have developed (see Fig. 7) and also linear ( $\mathrm{sp}^{1}$-bonded) chains. We may therefore term this process graphitization. For larger velocities, up to $1500 \mathrm{~m} \mathrm{~s}^{-1}$, the $\mathrm{sp}^{3}$ fraction increases again; this is caused by the increasingly high compressive pressure, which does not allow the formation of the low-coordinated adhesive neck (see Fig. 8). For even larger velocities, the $\mathrm{sp}^{3}$ fraction is reduced again; this case will be discussed in Sect. 3.4 below.

When averaged over the entire $\mathrm{sp}^{3}=55 \%$ system, the $\mathrm{sp}^{3}$ fraction stays roughly constant at around $53-55 \%$ up to a velocity of $v=1500 \mathrm{~km} \mathrm{~s}^{-1}$, demonstrating that the collision-induced hybridization changes only affect a small volume within the collision zone. At $3000 \mathrm{~m} \mathrm{~s}^{-1}$, however, the averaged $\mathrm{sp}^{3}$ fraction sinks to only $40 \%$ (see Sect. 3.4).

Figure 9 shows how these results depend on the NP radius. For the low-sp $\mathrm{sp}^{3}$ specimens, $\mathrm{sp}^{3}=20-40 \%$, the rise of the $\mathrm{sp}^{3}$ fraction with velocity is qualitatively similar, but not so pronounced as for the case of the large $R=20 \mathrm{~nm}$ spheres. In particular the $\mathrm{sp}^{3}=20 \%$ NPs with radius $R=6.5 \mathrm{~nm}$ show considerably less tendency to form $\mathrm{sp}^{3}$ bonds, reaching postcollision values of only $36-40 \%$. The $\mathrm{sp}^{3}=55 \%$ NPs also show a strong reduction in changing their hybridization in the small NPs; in the $R=6.5 \mathrm{~nm} \mathrm{NPs,} \mathrm{their} \mathrm{sp}^{3}$ fraction always stays above

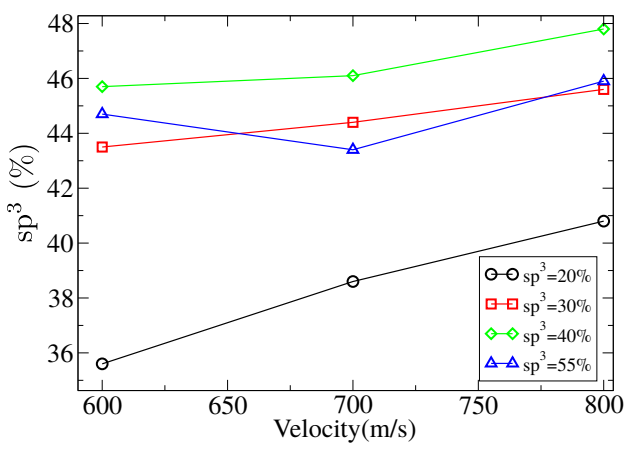

(a)

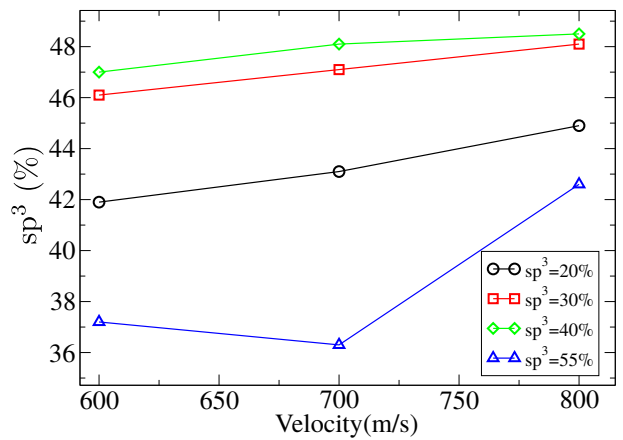

(b)

Fig. 9. Dependence of $\mathrm{sp}^{3}$ fraction in the contact region on collision velocity $v$ for NPs of radius (a) $6.5 \mathrm{~nm}$ and (b) $15 \mathrm{~nm}$.

43\%. The stronger effect in the larger NPs may be connected to the fact that the time during which the large compression lasts is roughly proportional to $R$, according to simple Hertz theory (Johnson 1985, Eq. (11.24)). Thus, in larger NPs, there is more time for the phase transformation to occur. On the other hand, we must also bear in mind that the true collision zone becomes smaller for smaller NP radii, while our analysis slab of $4 \mathrm{~nm}$ width has been kept unchanged. We note that even in these small spheres, the $\mathrm{sp}^{3}$ fraction shows a minimum at a velocity of $700 \mathrm{~m} \mathrm{~s}^{-1}$, showing that the formation of the filamentary adhesive neck also persists for these smaller NPs.

The unusual behavior of $\mathrm{sp}^{3}=55 \%$ NPs at a collision velocity of $v=700 \mathrm{~m} \mathrm{~s}^{-1}$ is emphasized in Fig. 10; at this velocity, NPs of all radii studied have the lowest $\mathrm{sp}^{3}$ fraction in the collision zone, indicating the formation of a strong filamentary neck. In addition, this figure shows that larger NPs lose more $\mathrm{sp}^{3}$ bonds in their collision zone, as their neck becomes broader with increasing NP radius $R$.

We can estimate the pressure $p$ arising during compression in the collision zone as follows. From the Hugoniot relationship we may derive the simple approximation (Millán et al. 2016a)

$p=\rho v c_{\mathrm{s}}$,

where $c_{\mathrm{s}}=(E / \rho)^{1 / 2}$ is the velocity of sound, which increases with $\mathrm{sp}^{3}$ content (Table 1). We evaluate Eq. (4) for a collision velocity of $v=600 \mathrm{~m} \mathrm{~s}^{-1}$. Since both the density and the velocity of sound increase with $\mathrm{sp}^{3}$, a strong increase in the collisioninduced pressure $p$ with $\mathrm{sp}^{3}$ content is the result (see Table 1).

The pressures estimated above for this collision velocity are much smaller than the elastic limits for pure diamond (around 


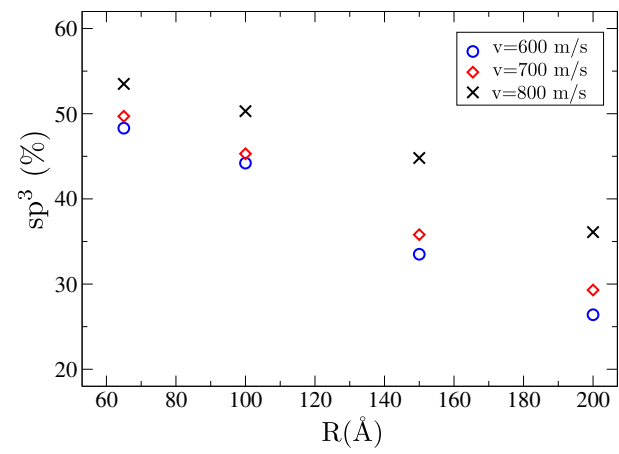

Fig. 10. Dependence of final $\mathrm{sp}^{3}$ fraction in the contact region on NP radius $R$ for NPs with $\mathrm{sp}^{3}=55 \%$ for various collision velocities.

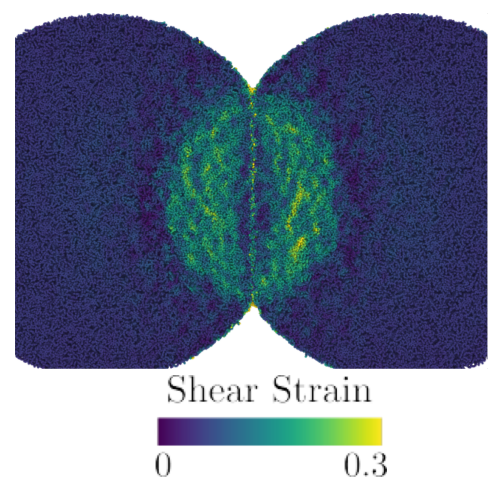

Fig. 11. Snapshots showing the conformation of two $R=20 \mathrm{~nm}$ NPs, $\mathrm{sp}^{3}=20 \%$, at a collision velocity of $v=800 \mathrm{~m} \mathrm{~s}^{-1}$, immediately after maximum compression. Atoms are colored according to their shear strain.

$150 \mathrm{GPa}$ Oleynik et al. 2008), and pure graphite (around $65 \mathrm{GPa}$ and $2 \mathrm{~km} \mathrm{~s}^{-1}$ piston velocity Pineau 2013). For a-C, using the Tersoff potential it was found that, for initial densities above $2.38 \mathrm{~g} \mathrm{~cm}^{-3}$, the shock behavior was elastic, until chemical changes started above $65 \mathrm{GPa}$ (Reed et al. 2010, Fig 6a). On the other hand, our pressure values of $12-25 \mathrm{GPa}$ are in the range of the experimental data for the phase transition from graphite to diamond in $\mathrm{C}$, which is on the order of $20 \mathrm{GPa}$ for defective materials, but higher for ideal crystals (Pineau 2013; Pineau et al. 2015). We conclude that the compressive pressures achieved during the collision are sufficient to trigger phase transformations in our NPs.

\subsection{Materials behavior}

Figure 11 illustrates the plastic deformation occurring in a collision of two $\mathrm{sp}^{3}=20 \% \mathrm{NPs}, R=20 \mathrm{~nm}$, at a collision velocity of $v=800 \mathrm{~m} \mathrm{~s}^{-1}$, immediately after maximum compression. Here, atoms are colored according to the strain they suffered during the collision. The strained region defines a quite clearly identifiable zone, which is of nearly spherical shape around the center of the contact plane with radius $a_{\mathrm{c}}$. Interestingly, this zone is not homogeneously sheared, but the highest shears appear in narrow regions, indicating the occurrence of localized shear transformation zones (STZs). Such STZs are known to govern the plastic deformation of amorphous material (Falk \& Langer 1998; Schuh \& Lund 2003; Huang et al. 2013); they were previously shown also to appear in energetic collisions of amorphous silica NPs (Nietiadi et al. 2017b). Here is the first time that their relevance is also demonstrated in a-C NP collisions. Their appearance may

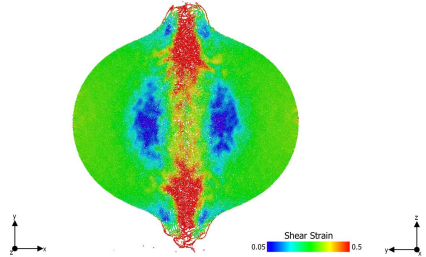

(a) (b)
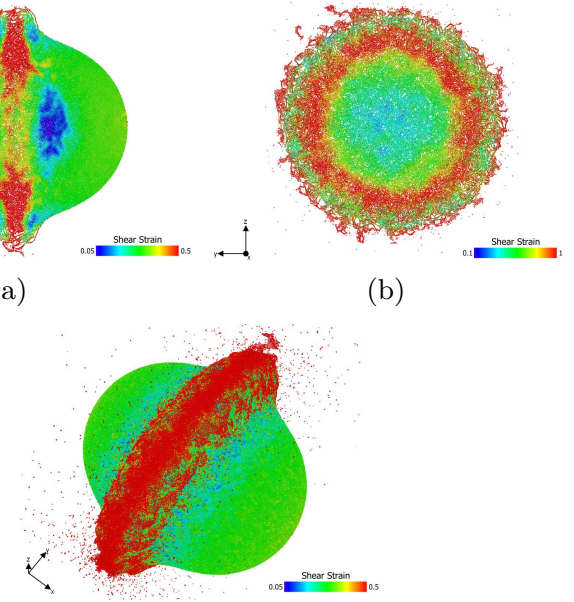

(c)

Fig. 12. Snapshots of the final configuration of two $R=20 \mathrm{~nm}$ NPs of $\mathrm{sp}^{3}=55 \%$ colliding with a velocity of $v=3000 \mathrm{~m} \mathrm{~s}^{-1}$. Crosssectional view $(a)$ parallel and $(b)$ perpendicular to the collision axis. (c) Perspective view. Atoms are colored according to their shear strain.

be significant for the future mechanical behavior of collided NPs since the material has been softened in these STZs, which might therefore be more susceptible to future cracking.

During collision, the temperature may reach high values in the collision zone. As an example, we obtained values of $3200 \mathrm{~K}$ at the time of maximum compression for $\mathrm{sp}^{3}=20 \%$ NPs with radius $R=20 \mathrm{~nm}$ colliding with a velocity of $v=800 \mathrm{~m} \mathrm{~s}^{-1}$. This high temperature is induced by the work done in the compression of the NPs. For $\mathrm{sp}^{3}=55 \%$ NPs under the same collision conditions, the temperature is considerably smaller $(1700 \mathrm{~K})$ in agreement with the larger bulk modulus of this specimen.

\subsection{High-velocity collision}

We also studied a collision at a high velocity, $v=3 \mathrm{~km} \mathrm{~s}^{-1}$. This was done for $\mathrm{sp}^{3}=55 \%$ NPs of radius $R=20 \mathrm{~nm}$. Figure 12 shows the final conformation of the merged NPs. The material in the contact plane expanded to form a bulge between the two merged NPs. We note that an increase in the contact radius beyond the values predicted by JKR was already visible at collision velocities of $600 \mathrm{~m} \mathrm{~s}^{-1}$ (see Fig. 5). This process has now become even more pronounced. It may be assumed that the high temperatures reached in the collision zone allowed for the material to expand so significantly.

The side view (Fig. 12a) confirms that the major strain occurred around the contact plane and in the bulge. However, the entire material underwent strains of around 0.25 , simply because the two spheres collapsed upon merging. The axial view (Fig. 12b) shows how strain monotonically increases from the collision axis toward the forming bulge. In all panels shown in Fig. 12, low-density material can be seen that has formed at the bulge.

We show a zoom in on this material in Fig. 13. Clearly, the emergence of several varieties of graphitic structures can be observed. These structures are very open, leaving room between them that make the structure very porous. It is known that high porosities change the behavior of NPs drastically in several respects, such as the thermal response (Okamoto et al. 1994) and also the dielectric constant (Mukai et al. 1992).

The strong roughening of the surface enhances the surface area of the merged NP. This may have consequences in a space 


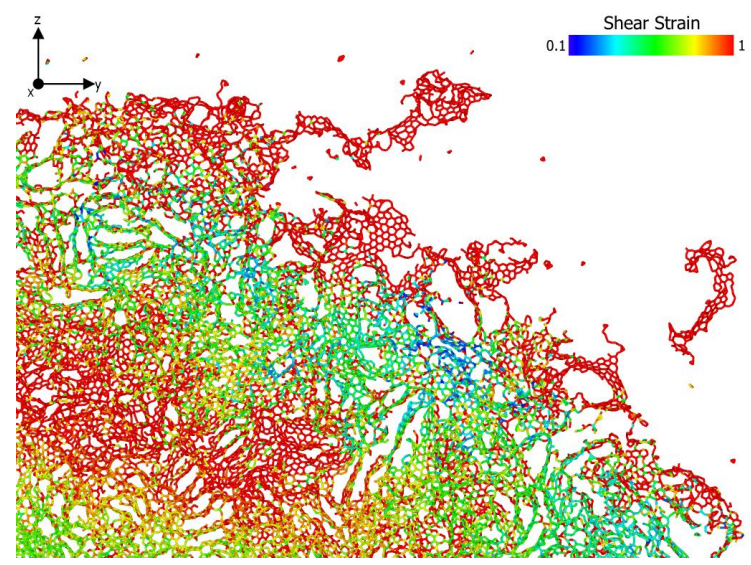

Fig. 13. Graphitic structures generated in the collision of two $R=20 \mathrm{~nm}$ NPs of $\mathrm{sp}^{3}=55 \%$. Zoom in on the collision-induced bulge created at a velocity of $v=3000 \mathrm{~m} \mathrm{~s}^{-1}$. Atoms are colored according to their shear strain.

environment for catalytic processes occurring on the surface and also for gas adsorption (Li \& Greenberg 2000). The rim of material coming out of the central collision zone is similar to the rim observed in the collision of diamond nanoparticles, $3 \mathrm{~nm}$ diameter, at $10 \mathrm{~km} \mathrm{~s}^{-1}$, simulated with ab-initio MD (Johnson et al. 2017). High shear and high temperature has been suggested as the cause of long linear carbon chains at the collision interface, somewhat akin to the collision scenario here at significantly lower velocities, but for a softer material.

Particle ejection becomes a major process at these high velocities (see Fig. 12c). A total of 80000 atoms - corresponding to $0.8 \%$ of the total material - have been ejected. Many of them appear in the form of clusters. Several of them can be spotted in Fig. 13. These are mainly based on graphitic material and may be denoted as PACs. The largest PAC contained 610 atoms.

\subsection{Discussion}

Light scattering from nanoparticles usually assumes several simplifications, like homogeneity and the fact that a dielectric function can be assigned to the whole nanoparticle. Some approaches for nanoparticles of up to a few nanometers assume only graphitic-like structures with up to a few thousand atoms (Li \& Draine 2001). In our simulations, for nanoparticles with few million atoms, the collision generates large inhomogeneities in chemical bonding inside the nanoparticle, and obtaining the resulting spectra would be difficult, because neither the quantum-based spectroscopy methods available for relatively few atoms, nor the continuum approaches used for $\mu \mathrm{m}$-sized particles could be used.

Granular-mechanics approaches have been often used to describe granular collisions. These microscopic approaches can be useful (Krijt et al. 2013), but due to their formulation they cannot describe changes in the internal structure of the grains, such as those found here, where bonding modifications inside the nanoparticles affect energy dissipation and sticking.

While the present study focuses on velocities of a few hundred meters per second and NP radii of at most $20 \mathrm{~nm}$, the collision outcome of carbon NPs at smaller velocities and larger radii will also be interesting for astrophysical applications. In analogy to other materials studied, it may be inferred that lower velocities would lead to sticking, and eventually would lead to sticking without significant chemical change; such collisions thus lead to trivial results. The simulation of larger NPs, on the other hand, is nowadays hardly possible due to the computational cost of using reactive interatomic potentials; actually, the simulations presented in this study are already pushing the limits of NP sizes that are possible with these simulations in mid-size computational clusters. However, we would speculate that larger $\mathrm{aC}$ NPs will behave similarly to the ones simulated here with respect to their phase transformation behavior, with plasticity appearing at even lower collision velocities (Millán et al. 2016b).

\section{Conclusions}

We employed MD simulations to study the effects of the collisions of a-C NPs. Our findings are for bare NPs as they might be found in space in the vicinity of a star where temperatures are so high as to let all adsorbates desorb. We find the following features.

1. In the velocity regime of $100-3000 \mathrm{~m} \mathrm{~s}^{-1}$, the collisions are always sticking. This is caused by the high density of dangling bonds at the surface, which produce a high surface energy. The correspondingly high attraction force prevents bouncing. As a consequence, carbon dust has a short collision-induced agglomeration time, since fragmentation events are missing.

2. In addition, the material phase transforms in the collision zone by converting $\mathrm{sp}^{2}$ to $\mathrm{sp}^{3}$ material. This occurs for $\mathrm{sp}^{3}$-poor material $\left(\mathrm{sp}^{3} \leq 40 \%\right)$ and produces material with $\mathrm{sp}^{3}>50 \%$. This process occurs during the collision-induced compression, which locally increases the coordination number and hence the $\mathrm{sp}^{3}$ content. The process is akin to the pressure-induced phase transformation from graphite to diamond. Such hybridization changes are bound to change the IR spectra of NPs.

3. Only for the highest $\mathrm{sp}^{3}$ fraction, $55 \%$, is a different behavior observed, since there - immediately at the contact surface $-\mathrm{sp}^{1}$ filaments and $\mathrm{sp}^{2}$ graphitic sheets are produced. This process of "graphitization" occurs in the expanding phase of the collision; the resulting material acts as a glue to keep the NPs contacted.

4. Macroscopic JKR theory describes the ingoing trajectory of the two colliding grains. However, the contact radius reached at maximum compression is larger in the simulation than in JKR theory, pointing again to the dominant role of attractive intergranular forces.

5. Apart from the importance of phase transformation, sheartransformation zones could be identified in the collision zone that are responsible for the NP plasticity.

6. At the highest collision velocity studied, $3 \mathrm{~km} \mathrm{~s}^{-1}$, an intergranular bulge formed, which showed a high degree of graphitization, as well as porosity. These features, as well as the observed surface roughening, have consequences for the thermal and optical properties of the NPs, and also for their potential for gas adsorption and surface catalytic reactions. In addition, ejection of material from the NPs was observed, both in the form of atoms and PACs.

Passivation of the surface, for instance by adsorbed $\mathrm{H}$, may have a strong effect on the collision behavior. For the analogous case of silica NP collisions, it was shown that surface passivation reduces the surface energy by around one order of magnitude (Kimura et al. 2015; Nietiadi et al. 2020). However, the simulation of collisions of surface-passivated or even hydrogenated a-C NPs is not straightforward, since a reliable interatomic potential, which represents both the $\mathrm{C}-\mathrm{H}$ chemistry and the high-pressure phase transformations faithfully, does not appear to be available. The REAX potential (Srinivasan et al. 2015), which possibly 
best presents this option, is unfortunately computationally quite expensive.

The present study explored the phenomena occurring in carbon-grain collisions in a very restricted model scenario - limited size range in the nanometer range $(R \leq 20 \mathrm{~nm})$, pure carbon with $\mathrm{sp}^{3}$ fractions between 20 and $55 \%$, only central collisions - and can therefore not be considered as a comprehensive study. Rather it aims at exploring which phenomena may occur in this class of materials, which has up to now been little investigated. Indeed we find that (i) the $\mathrm{sp}^{3}$ fraction is an important material quantity that dictates the elastic and adhesive properties of the grains; (ii) collisions induce phase transformations in the grain that alter their properties. Collision-processed grains may have surface properties that differ from those with which they were generated. This not only applies to the mechanical properties, but may also affect the optical properties, since it is known that refractive indices and extinction coefficients of amorphouscarbon grains depend on their $\mathrm{sp}^{3}$ fraction (Koike et al. 1980; Zubko et al. 1996; Hiratsuka et al. 2013).

Acknowledgements. Collision simulations were performed at the High Performance Cluster Elwetritsch (Regionales Hochschulrechenzentrum, TU Kaiserslautern, Germany). We acknowledge financial support by the Deutsche Forschungsgemeinschaft within project Ur 32/27-2. F.V. and R.G. thank the Fondo Nacional de Investigaciones Científicas y Tecnológicas (FONDECYT, Chile) under grants \#11190484, and \#11180557, and Financiamiento Basal para Centros Científicos y Tecnológicos de Excelencia AFB180001. E.M.B. acknowledges funding from a SIIP-UNCuyo grant 06/M104. This research was partially supported by the supercomputing infrastructure of the NLHPC (ECM-02)

\section{References}

Abdu, Y. A., Hawthorne, F. C., \& Varela, M. E. 2018, ApJ, 856, L9

Brilliantov, N. V., Albers, N., Spahn, F., \& Pöschel, T. 2007, Phys. Rev. E, 76, 051302

Charitidis, C. A. 2010, Int. J. Refract. Metals Hard Mater., 28, 51

Dai, Z. R., Bradley, J. P., Joswiak, D. J., et al. 2002, Nature, 418, 157

De La Pierre, M., Bruno, M., Manfredotti, C., et al. 2014, Mol. Phys., 112, 1030

de Tomas, C., Suarez-Martinez, I., \& Marks, N. A. 2016, Carbon, 109, 681

de Tomas, C., Aghajamali, A., Jones, J. L., et al. 2019, Carbon, 155, 624

Derjaguin, B. V., Muller, V. M., \& Toporov, Y. P. 1975, J. Colloid Interface Sci., 53,314

Dimitrijevic, M. S. 2005, Proc. IV Serbian-Bulgarian Astronomical Conference, eds. M. S. Dimitrijevic, V. Golev, L. C. Popovic, \& M. Tsvetkov, 51

Dominik, C., \& Tielens, A. G. G. M. 1997, ApJ, 480, 647

Draine, B. T. 2003, Annu. Rev. Astron. Astrophys., 41, 241

Falk, M. L., \& Langer, J. S. 1998, Phys. Rev. E, 57, 7192

Ferrari, A. C., Kleinsorge, B., Morrison, N. A., et al. 1999a, J. Appl. Phys., 85, 7191

Ferrari, A. C., Robertson, J., Beghi, M. G., et al. 1999b, Appl. Phys. Lett., 75 1893

Goto, M., Henning, T., Kouchi, A., et al. 2009, ApJ, 693, 610

He, H., \& Thorpe, M. F. 1985, Phys. Rev. Lett., 54, 2107

Hiratsuka, M., Nakamori, H., Kogo, Y., et al. 2013, J. Solid Mech. Mater. Eng., 7,187

Huang, P. Y., Kurasch, S., Alden, J. S., et al. 2013, Science, 342, 224

Jana, R., Savio, D., Deringer, V. L., \& Pastewka, L. 2019, Modell. Simul. Mater. Sci. Eng., 27, 085009

Johnson, K. L. 1985, Contact Mechanics (Cambridge: Cambridge University Press)

Johnson, K. L., Kendall, K., \& Roberts, A. D. 1971, Proc. R. Soc. London. Ser. A, 324,301

Johnson, D. F., Mullin, J. M., \& Mattson, W. D. 2017, J. Phys. Chem. C, 121, 1140

Kalweit, M., \& Drikakis, D. 2006, Phys. Rev. B, 74, 235415

Kelires, P. C. 1994, Phys. Rev. Lett., 73, 2460

Kern, G., \& Hafner, J. 1997, Phys. Rev. B, 56, 4203

Kimura, H., Wada, K., Senshu, H., \& Kobayashi, H. 2015, ApJ, 812, 67

Koike, C., Hasegawa, H., \& Manabe, A. 1980, Astrophys. Space Sci., 67, 495

Krijt, S., Güttler, C., Heißelmann, D., Dominik, C., \& Tielens, A. G. G. M. 2013, J. Phys. D, 46, 435303

Lewis, R. S., Anders, E., \& Draine, B. T. 1989, Nature, 339, 117
Li, A., \& Draine, B. T. 2001, ApJ, 554, 778

Li, A., \& Greenberg, J. M. 2000, in NATO Science Series II: Mathematics, Physics and Chemistry, Solid state astrochemistry, eds. V. Pirronello, J. Krelowski, \& G. Manicò (Dordrecht: Kluwer Academic Publishers), 120, 37

Li, L., Xu, M., Song, W., et al. 2013a, Appl. Surf. Sci., 286, 287

Li, Z., Wang, Y., Kozbial, A., et al. 2013b, Nat. Mater., 12, 925

Li, X., Wang, A., \& Lee, K.-R. 2018, Comput. Mater. Sci., 151, 246

Liew, K. M., He, X. Q., \& Wong, C. H. 2004, Acta Mater., 52, 2521

Liu, C., Lin, Y., Zhou, Z., \& Li, K.-Y. 2017, Carbon, 122, 276

Marks, N. A. 2000, Phys. Rev. B, 63, 035401

Marks, N. A., Cooper, N. C., McKenzie, D. R., et al. 2002, Phys. Rev. B, 65, 075411

Marks, N. A., Lattemann, M., \& McKenzie, D. R. 2012, Phys. Rev. Lett., 108, 075503

Mathis, J. S., Rumpl, W., \& Nordsieck, K. H. 1977, ApJ, 217, 425

Maugis, D. 2000, Contact, Adhesion and Rupture of Elastic Solids (Berlin: Springer)

Micelotta, E. R., Jones, A. P., Cami, J., et al. 2012, ApJ, 761, 35

Millán, E. N., Tramontina, D. R., Urbassek, H. M., \& Bringa, E. M. 2016a, Phys. Chem. Chem. Phys., 18, 3423

Millán, E. N., Tramontina, D. R., Urbassek, H. M., \& Bringa, E. M. 2016b, Phys. Rev. E, 93, 063004

Mukai, T., Ishimoto, H., Kozasa, T., Blum, J., \& Greenberg, J. M. 1992, A\&A, 262,315

Nietiadi, M. L., Umstätter, P., Alabd Alhafez, I., et al. 2017a, Geophys. Res. Lett., 44,822

Nietiadi, M. L., Umstätter, P., Tjong, T., et al. 2017b, Phys. Chem. Chem. Phys., 19,16555

Nietiadi, M. L., Millán, E. N., Bringa, E. M., \& Urbassek, H. M. 2019, Phys. Rev. E, 99, 032904

Nietiadi, M. L., Rosandi, Y., \& Urbassek, H. M. 2020, Nanoscale Res. Lett., 15, 67

Nittler, L. R. 2003, Earth Planet. Sci. Lett., 209, 259

Nuth, J. A. 1987, Nature, 329, 589

Ohnishi, N., Bringa, E. M., Remington, B. A., et al. 2008, J. Phys. Conf. Ser., 112,042017

Okamoto, H., Mukai, T. \& Kozasa, T. 1994, Planet. Space Sci., 42, 643

Oleynik, I. I., Landerville, A. C., Zybin, S. V., Elert, M. L., \& White, C. T. 2008, Phys. Rev. B, 78, 180101

Ormel, C. W., Paszun, D., Dominik, C., \& Tielens, A. G. G. M. 2009, A\&A, 502, 845

Panella, B., Hirscher, M., \& Roth, S. 2005, Carbon, 43, 2209

Pineau, N. 2013, J. Phys. Chem. C, 117, 12778

Pineau, N., Soulard, L., Colombet, L., et al. 2015, J. Appl. Phys., 117, 115902

Plimpton, S. 1995, J. Comput. Phys., 117, 1

Reed, E. J., Maiti, A., \& Fried, L. E. 2010, Phys. Rev. E, 81, 016607

Schuh, C. A., \& Lund, A. C. 2003, Nat. Mater., 2, 449

Schwan, J., Ulrich, S., Roth, H., et al. 1996, J. Appl. Phys., 79, 1416

Srinivasan, S. G., van Duin, A. C. T., \& Ganesh, P. 2015, J. Phys. Chem. A, 119, 571

Stukowski, A. 2010, Model. Simul. Mater. Sci. Eng., 18, 015012

Suk, J. W., Murali, S., An, J., \& Ruoff, R. S. 2012, Carbon, 50, 2220

Sun, W., Zeng, Q., \& Yu, A. 2015, Chem. Eng. Sci., 121, 23

Sun, W., Jiang, J., \& Chen, P. 2020, Powder Technol., 364, 689

Tanaka, H., Wada, K., Suyama, T., \& Okuzumi, S. 2012, Prog. Theor. Phys. Suppl., 195, 101

Tersoff, J. 1988, Phys. Rev. Lett., 61, 2879

Thornton, C., \& Ning, Z. 1998, Powder Technol., 99, 154

Tielens, A. G. G. M. 2005, The Physics and Chemistry of the Interstellar Medium (Cambridge, UK: Cambridge University Press)

Tielens, A. 2008, ARA\&A, 46, 289

Tielens, A. G. G. M., McKee, C. F., Seab, C. G., \& Hollenbach, D. J. 1987, ApJ, 319, L109

Vis, R. D., Mrowiec, A., Kooyman, P. J., Matsubara, K., \& Heymann, D. 2002, Meteorit. Planet. Sci., 37, 1391

Wang, Z., \& Yin, J. 1998, Chem. Phys. Lett., 289, 189

Wickramasinghe, N. C., Wickramasinghe, A. N., \& Hoyle, F. 1999, Astrophys. Space Sci., 268, 289

Wolf, D. 1990, Surf. Sci., 226, 389

Yang, W., Mao, S., Yang, J., et al. 2016, Sci. Rep., 6, 24187

Yang, W., Yang, J., Dong, Y., et al. 2018, Carbon, 137, 411

Zhang, H., Duan, Z., Zhang, X., et al. 2013, Phys. Chem. Chem. Phys., 15, 11794

Zhang, H., Zhou, L., Noonan, O., et al. 2014, Adv. Funct. Mater., 24, 4337

Zubko, V. G., Mennella, V., Colangeli, L., \& Bussoletti, E. 1996, in The Role of Dust in the Formation of Stars, eds. H. U. Käufl, \& R. Siebenmorgen (Berlin: Springer ), 333 